Chapman University

Chapman University Digital Commons

Psychology Faculty Articles and Research

Psychology

2013

\title{
Placental Corticotropin-Releasing Hormone Mediates the Association Between Prenatal Social Support and Postpartum Depression
}

Jennifer Hahn-Holbrook

Chapman University, hahnholb@chapman.edu

Christine Dunkel Schetter

University of California - Los Angeles

Chander Arora

Cedars Sinai Medical Center

Calvin J. Hobel

Cedars Sinai Medical Center

Follow this and additional works at: http://digitalcommons.chapman.edu/psychology_articles

Part of the Biological Psychology Commons, Maternal and Child Health Commons, Mental and Social Health Commons, and the Women's Health Commons

\section{Recommended Citation}

Hahn-Holbrook, J., Dunkel Schetter, C., Chander, A., \& Hobel, C. (2013). Placental corticotropin-releasing hormone mediates the association between prenatal social support and postpartum depression. Clinical Psychological Science, 1, 253-265.

doi: $10.1177 / 2167702612470646$ 


\section{Placental Corticotropin-Releasing Hormone Mediates the Association Between Prenatal Social Support and Postpartum Depression}

\section{Comments}

This is a pre-copy-editing, author-produced PDF of an article accepted for publication in Clinical Psychological Science, volume 1, 2013 following peer review. The definitive publisher-authenticated version is available online at < a href="http://dx.doi.org/10.1177/2167702612470646">DOI: 10.1177/2167702612470646.

\section{Copyright}

Sage 


\title{
Placental Corticotropin-Releasing Hormone Mediates the Association Between Prenatal Social Support and Postpartum Depression
}

\author{
Jennifer Hahn-Holbrook ${ }^{1}$, Christine Dunkel Schetter ${ }^{1}$, Chander Arora ${ }^{2}$, and Calvin J. \\ Hobel ${ }^{1,2}$ \\ ${ }^{1}$ Department of Psychology, University of California, Los Angeles, California \\ ${ }^{2}$ Cedars-Sinai Medical Center, Los Angeles, California
}

\begin{abstract}
Three decades of research point to both biological and psychological risk factors for postpartum depression, but very little research integrates the two. This study bridged this gap by testing whether prenatal social support predicted depressive symptoms at 8 weeks postpartum in a multiethnic sample of 210 women and whether the stress hormone placental corticotropinreleasing hormone (pCRH), measured at 19, 29, and 37 weeks' gestation, mediated this relationship. We found that prenatal family support predicted significantly fewer depressive symptoms postpartum and more gradual increases in pCRH from 29 to 37 weeks' gestation. Furthermore, steeper increases in pCRH during this same period predicted more depressive symptoms postpartum. Finally, these changes in pCRH in late pregnancy mediated the relationship between prenatal family support and postpartum depressive symptoms. These results suggest that social and biological risk factors for postpartum depressive symptoms are intertwined and move us closer to an integrated biopsychosocial understanding of postpartum depression.
\end{abstract}

\section{Keywords}

corticotropin-releasing hormone; postpartum depression; social support; hypothalamic-pituitaryadrenal axis; placental-maternal pituitary-adrenal axis

\begin{abstract}
Depression costs the American economy approximately $\$ 81$ billion annually (Greenberg et al., 2003) and is the leading cause of disability in women (Nobel, 2005). Research suggests that women are especially prone to mood disorders during the postpartum period (Gotlib, Whiffen, Mount, Milne, \& Cordy, 1989; Kessler, 2003; Vesga-Lopez et al., 2008; Wisner, Peindel, \& Hanusa, 1993). As many as $85 \%$ of women will experience some mood disturbance postpartum (Stein, Marsh, \& Morton, 1981), manifesting in various forms. Conservative estimates suggest that $26 \%$ of women will experience a transient set of depressive-like symptoms typically occurring within 10 days after childbirth, often referred to as postpartum blues (Harris et al., 1994; O’Hara, Schlechte, Lewis, \& Wright, 1991; O’Hara, Zekoski, Philipps, \& Wright, 1990; Okano \& Nomura, 1992; Pitt, 1973). Approximately one in five women will experience a depressive episode within the first year
\end{abstract}

(C) The Author(s) 2013

Corresponding Author: Jennifer Hahn-Holbrook, Department of Psychology, University of California, Los Angeles, Box 951563, 1282 Franz Hall, Los Angeles, CA 90025-1563, jhahn@ psych.ucla.edu, dunkel@ psych.ucla.edu.

Declaration of Conflicting Interests

The authors declared that they had no conflicts of interest with respect to their authorship or the publication of this article. 
after birth (Gaynes et al., 2005), with the majority of cases occurring within the initial 12 weeks after delivery (O'Hara \& Swain, 1996). Depression and postpartum depression are not considered distinct disorders by the fourth edition of the Diagnostic and Statistical Manual of Mental Disorders; rather, postpartum depression is defined as an episode of major depressive disorder that occurs within 4 weeks postpartum (American Psychiatric Association, 1994). Depression in new mothers is especially damaging because it can impair parenting behaviors (Field, 2010; Paulson, Dauber, \& Leiferman, 2006) and result in longterm negative consequences for the cognitive, emotional, and behavioral development of their children (see Grace, Evindar, \& Stewart, 2003, for a review). In addition, maternal distress and depression adversely affect other family relationships and are associated with marital discord (Zelkowitz \& Milet, 1996). Reflective of its prevalence and seriousness, postpartum depression has received considerable attention over the past three decades.

Clinical science has not neglected the study of postpartum depression. A huge literature has grown concerning its definition, measurement, consequences, treatment, and etiological predictors. Despite this large repository of knowledge on postpartum depression, it is noteworthy that very little research has been integrative in the sense that it synthesizes information across disciplines (for exceptions, see Halbreich, 2005; Ross, Sellers, Gilbert Evans, \& Romach, 2004; Roy, Evers, Avison, \& Campbell, 2010). For example, psychological studies laudably highlight psychosocial predictors, such as life stress, marital quality, or social support (see C. T. Beck, 2001; O'Hara, 2009, for reviews), but rarely examine biological variables, and when they do, they often fail to investigate psychobiological interactions or synergies. Conversely, biological investigations rightly focus on risk factors such as gonadal or stress hormone dysregulation in pregnancy (see Bloch, Daly, \& Rubinow, 2003; Hillerer, Neumann, \& Slattery, 2012; Zonana \& Gorman, 2005 , for reviews) but are largely heedless of the influence of psychosocial factors on these biological mechanisms. The traditional division between psychological and biological factors can be heuristically useful in isolating different predictors of postpartum depression, but this practice should not be allowed to obscure the ultimate scientific goal of interdisciplinary integration.

The primary goal of the present work was to bring together the disparate psychological and biological literatures on postpartum depression by examining the interrelations between a psychosocial resource and a neuroendocrine risk factor implicated in the disorder. The psychological resource examined was the amount of social support that expecting mothers perceived was available from family and the baby's father during pregnancy, and the neuroendocrine factor studied was corticotropin-releasing hormone of placental origin $(\mathrm{pCRH})$ released and measurable in the maternal bloodstream. Given the importance of social support in buffering women from stress hormone dysregulation in the nonpregnant state (see Ehlert, Gaab, \& Heinrichs, 2001; Pariante \& Lightman, 2008, for reviews) and the emerging evidence linking pCRH in pregnancy to postpartum depression (Yim et al., 2009), we sought to combine these two lines of evidence by hypothesizing that social support would protect women against postpartum depressive symptoms, in part, by mitigating deleterious increases in pCRH during pregnancy. Thus, we attempt to replicate the landmark finding by Yim and colleagues (2009) demonstrating that higher pCRH in pregnancy is a risk factor for postpartum depression with a larger, more ethnically diverse sample, but we also attempt to extend this line of research by testing whether pCRH is a potential biological mediator between social support and reduced risk of postpartum depression.

Weak or absent social support has long been recognized as a consistent risk factor for postpartum depression (C. T. Beck, 1996, 2001; O'Hara, 2009; O'Hara \& Swain, 1996; Robertson, Grace, Wallington, \& Stewart, 2004). A meta-analysis of five studies found that poor social support during pregnancy exerted a large to moderate effect on risk of 
postpartum depression (delta $=-0.63 ; 95 \%$ confidence interval: $-0.75,-0.51 ; r=-0.30$; O'Hara \& Swain, 1996). Controlled longitudinal studies have documented, for example, that women who have smaller social networks or who report poor support from family or the baby's father during pregnancy are more likely to develop postpartum depression (Collins, Dunkel-Schetter, Lobel, \& Scrimshaw, 1993; Logsdon \& McBride, 1994). Although social support comes in many forms, perceived support—defined as the subjective feeling that one is loved and cared for and can count on others when in need-may be especially protective against maternal depression (Ramona \& Ferketich, 1988). One pathway through which perceived support protects against depression is its role in mitigating the effects of stress (Cohen \& Wills, 1985). For example, a multiethnic study of 192 mothers found that lowincome women who reported relatively more perceived support from the baby's father also reported less anxiety about their pregnancies and less overall stress and were less likely to have depressive symptoms postpartum (Norbeck \& Anderson, 1989). Social support has also been shown to dampen biological stress responses in the nonpregnant state (see Uchino, Cacioppo, \& Kiecolt-Glaser, 1996, for a review). A search of the literature produced no investigations testing whether social support is linked to lower stress hormone levels during pregnancy. Extension of this research to pregnancy could provide a key link between psychosocial theories of postpartum depression involving the central role of social support and biological research implicating stress hormone exposure in pregnancy.

Depressive disorders have been conceptualized as involving dysregulation of the body's stress response systems such that affective and biological stress responses occur in disproportion to events or persist for extended periods resulting in negative mood, cognitive difficulties, heightened anxiety, and physiological dysregulation (Ehlert et al., 2001; Pariante \& Lightman, 2008). This profile implicates involvement of the hypothalamicpituitary-adrenal (HPA) axis, a complex biological system that activates under stress exposure and enables the organism to meet the demands of the stressor by increasing energy and vigilance toward potential threats. The release of corticotropin-releasing hormone $(\mathrm{CRH})$ from the hypothalamus starts the HPA axis cascade and triggers the release of adrenocorticotropic hormone (ACTH) from the pituitary into the peripheral bloodstream. In turn, the release of ACTH triggers cortisol to be released from the adrenal glands into the bloodstream. Cortisol adjusts its own further activity through a negative feedback system, downregulating the HPA axis response by inhibiting the production of CRH in the hypothalamus and ACTH in the pituitary (S. M. Smith \& Vale, 2006). If the HPA axis is activated too often or too acutely, this system can become dysregulated in the sense that it resists returning to homeostasis (for reviews, see McEwen, 1998; Nestler et al., 2002). Many people with depression show evidence of dysregulated HPA axis activity that remits with treatment (Arborelius, Owens, Plotsky, \& Nemeroff, 1999; Gold, Licinio, Wong \& Chrousos 1995; Holsboer, 2001). Depressive states have also been linked to elevated levels of CRH in the brain (Arborelius et al., 1999; Holsboer, 2001; Kasckow, Baker, \& Geracioti, 2001). Studies with rodents and primates highlight the striking parallels between symptoms of severe depression and the effects of centrally administered CRH. These include increased anxiety and fearfulness, decreased appetite, decreased sexual behavior, and increased heart rate and blood pressure (Arborelius et al., 1999; Chrousos, Torpy, \& Gold, 1998).

These links between symptoms of depression and HPA dysregulation may illuminate how the dramatic shifts in stress hormones characteristic of pregnancy and the postpartum precipitate depressive disorders related to childbearing. The placenta functions as an endocrine organ in pregnancy (Evain-Brion \& Malassine, 2003) and releases pCRH into the maternal bloodstream at levels from 60 to 700 times higher than those observed prior to pregnancy (Campbell et al., 1987). pCRH is detectable in the maternal bloodstream because levels become extremely high, unlike CRH released from the hypothalamus in the nonpregnant state (Sasaki et al., 1984; Shibasaki, Odagiri, Shizume, \& Ling, 1982). Thus, 
pregnancy affords researchers with a unique opportunity to study $\mathrm{CRH}$ without having to perform invasive procedures. $\mathrm{CRH}$ produced by the hypothalamus and that by the placenta have similar structures and actions on CRH receptors (Florio, Zatelli, Reis, degli Uberti, \& Petraglia, 2007; Petraglia, Florio, Nappi, \& Genazzani, 1996), but they play opposite roles in HPA axis activity. Recall that the hypothalamus decreases CRH production in response to cortisol, which leads to a downregulation of HPA axis activity. By contrast, placental tissue exposed to cortisol actually upregulates the production of pCRH. This flip causes pCRH levels to increase exponentially over the course of pregnancy, along with more modest increases in other HPA products, such as cortisol and ACTH (McLean, Thompson, Zhang, Brinsmead, \& Smith, 1994; Sasaki et al., 1984). This positive feedback loop in pCRH comes to a sudden halt at birth when the placenta is expelled from the body and HPA hormones drop to prepregnancy levels within a matter of days. The postpartum period can therefore be considered a time of readjustment by the HPA axis to placental CRH withdrawal (Hochberg, Pacak, \& Chrousos, 2003). Abnormally high levels of pCRH in pregnancy, followed by their subsequent, precipitous withdrawal, have been hypothesized to trigger postpartum depression in vulnerable individuals (Chrousos et al., 1998; Halbreich, 2005; Hochberg et al., 2003; Magiakou et al., 1996; Vitoratos, Papatheodorou, Kalantaridou, \& Mastorakos, 2006).

Despite the wider literature linking HPA axis dysregulation to depression in the nonpregnant state, only a few studies have investigated whether pCRH shifts during pregnancy pose a risk factor for postpartum depression. In a landmark work, Yim and colleagues (2009) found that mothers who had significantly accelerated pCRH trajectories between 23 and 26 weeks of gestation and significantly higher pCRH levels from the 18th week through the end of their pregnancies were more likely to show depressive symptoms at 9 weeks postpartum. Other researchers, however, have found associations between pCRH and depressive symptoms in pregnancy only (Meltzer-Brody et al., 2011; Rich-Edwards et al., 2008; Susman et al., 1999) or reported no association between pCRH and depressive symptoms (R. Smith et al., 1990), although timing and measurement issues may explain these inconsistencies, as discussed later.

We attempted to replicate and extend the results of Yim and colleagues (2009) suggesting that pCRH levels in pregnancy predict depressive symptoms postpartum. We hypothesized that social support during pregnancy would reduce the increases in pCRH over gestation and that this, in turn, would be protective against depressive symptoms postpartum. Thus, three primary hypotheses were tested: First, prenatal social support from the baby's father or family will predict lower levels of pCRH during pregnancy; second, increases in maternal pCRH over gestation will predict increased depressive symptoms postpartum; and third, pCRH will mediate the relationship between social support during pregnancy and depression symptoms postpartum. These hypotheses were tested in a longitudinal study of biopsychosocial stress processes in pregnancy and the postpartum.

\section{Methods}

\section{Participants}

Participants were 210 women from the Behavior in Pregnancy Study (BIPS; for full methods, see Hobel, Dunkel-Schetter, \& Roesch, 1998; Hobel, Dunkel-Schetter, Roesch, Castro, \& Arora, 1999). The BIPS recruited 688 women who were receiving care in prenatal clinics and private practices in hospital and managed care settings and who gave birth to live-born infants in hospitals in Los Angeles, California. To be eligible for the BIPS, women had to be English or Spanish speaking, have a singleton intrauterine pregnancy, and be under 20 weeks gestation at the time of recruitment. Women were included in this study if they had pCRH data for at least one time point during pregnancy, perceived social support 
data, and data on prenatal and postpartum depressive symptoms. Compared to participants in the larger study, women in this subsample were slightly older $(B=.04, S E=.02, p<.05)$, had somewhat higher incomes $(B=.096, S E=.041, p<.05)$, were more likely to be having their first child $(B=-.54, S E=.21, p<.01)$, and had slightly higher birth weight infants $(B$ $=.001, S E=.00, p<.01)$. This subsample did not differ significantly from the whole sample in terms of smoking in pregnancy, marital status, education, or preterm delivery.

The ethnic composition of this sample was 37\% Latina/ Hispanic, 29\% non-Hispanic White, $28 \%$ African American/ Black, and 4\% Asian/Pacific Islander, paralleling the demographics of the research sites. Seventy-seven percent of the sample was married or cohabitating with the baby's father at the time of the study. The mean age was 28.5 years $(S D=5.6)$, and the average household income fell in the range of $\$ 30,000$ to $\$ 40,000$ a year. Overall, this is a diverse and low- to middle-income sample of women with variation in father presence.

\section{Procedure}

Participants were recruited into the study before the 20th week of gestation and were followed at three time points during pregnancy (T1: $M=19$ weeks, range $=18-21 ; \mathrm{T} 2: M=$ 29 weeks, range $=27-31$ weeks; T3: $M=37$ weeks, range $=36-38$ weeks) and, on average, at 8 weeks postpartum (T4: range $=6-10$ weeks). $\mathrm{pCRH}$ was measured at each of the three prenatal assessments in maternal peripheral blood, drawn by a research nurse or phlebotomist. At the second prenatal assessment, women were interviewed by a trained project interviewer in their preferred language (English or Spanish [ $8 \%$ of this sample]) regarding the social support they perceived from the baby's father and their families.

Women were asked to fill out a depression screening instrument at T2 and T4. The intention behind using self-administration for the depression screener was to provide participants with additional anonymity in the hopes that it would encourage them to be more honest when answering questions about sensitive topics, although anyone requiring assistance was administered the screener orally by an interviewer.

\section{Measures}

Perceived social support-An eight-item scale used in past research (Turner, Frankel, \& Levin, 1983; Turner, Grindstaff, \& Phillips, 1990) assessed perceived social support from the baby's father during pregnancy. Items assessed the extent to which the mother perceived that the baby's father was affectionate, was understanding of her feelings, talked and spent time with her, could be counted on to provide financial support and to be there when needed, and would help when the baby came. The response scales ranged from 1 (strongly disagree) to 4 (strongly agree), and an index of perceived support from the baby's father was computed by averaging the scores on the eight items. The scale had good internal consistency (Cronbach's alpha $=.92)$.

A similar seven-item scale was used to assess perceived available support from family during pregnancy. This scale was adapted from the Provisions of Social Relations Scale (Turner et al., 1983) with the addition of one item concerning financial assistance. Items assess the extent to which the mother felt that her family could be relied on when needed, believed in her, would provide financial assistance if needed, and would provide help if she had problems. Participants rated the items on a 4-point Likert scale ranging from 1 (strongly disagree) to 4 (strongly agree). This scale also had high internal consistency (Cronbach's alpha $=.89)$. Both measures of social support have been used in pregnancy research and have been shown to predict birth and mental health outcomes (Collins et al., 1993; Feldman, Dunkel-Schetter, Sandman, \& Wadhwa, 2000). 
Depressive symptoms-The Beck Depression Inventory (BDI; A. X. Beck, Ward, Mendelson, Mock, \& Erbaugh, 1961) was used to assess depressive symptoms during pregnancy and postpartum. This widely used 21 -item scale assesses the cognitive, affective, and somatic components of depressive symptomatology. The index score can be used as a continuous variable to assess depressive symptoms, or cutoff scores can be used to distinguish mild depressive symptomatology (BDI score $\geq 10$ ) or moderate to severe depressive symptomatology (BDI score 219 ). In both psychiatric and student samples, the BDI cutoff scores have shown high convergent validity with psychiatric ratings of depression severity (A. X. Beck et al., 1961; Bumberry, Oliver, \& McClure, 1978; see Gotlib \& Cane, 1989, for a review). The BDI has also been validated for use in pregnant women (Holcomb, Stone, Lustman, Gavard, \& Mostello, 1996). The sensitivity of the BDI (i.e., the ability of the BDI to detect episodes of depression when present) is $100 \%$ for the $9 / 10$ cutoff and $47.6 \%$ for the $18 / 19$ cutoff. The specificity of the BDI (i.e., the ability of the BDI to detect the absence of depression when there is none present) is $86 \%$ for the $9 / 10$ cutoff and $98.6 \%$ for the 18/19 cutoff (Oliver \& Simmons, 1984).

Stress hormone measurement: pCRH-The protocol used to measure pCRH parallels that recommended by Latendresse and Ruiz (2008). At each time point during pregnancy, blood was collected in chilled glass tubes containing EDTA $(1 \mathrm{mg} / \mathrm{mL}$ of blood $)$ and aprotinin $\left(500 \mathrm{kIU} / \mathrm{mL}\right.$ of blood) and centrifuged at $4{ }^{\circ} \mathrm{C}$. Plasma was then stored at $-70^{\circ} \mathrm{C}$ until extraction. Plasma was thawed and then extracted with Sep-Pak C-18 cartridges (Waters Associates, Milford, MA) for measurement of immunoreactive pCRH levels. Acidified plasma was loaded onto columns previously activated with $60 \%$ acetonitrile in $1 \%$ trifluoroacetic acid. The columns were washed twice with $3 \mathrm{~mL}$ of $1 \%$ trifluoroacetic acid. The absorbed acid buffer and eluant dried in a speed vacuum concentrator (Savant Instruments, Micksville, NY). The dried extracts were stored at $-80^{\circ} \mathrm{C}$ and resuspended in radioimmunoassay buffer at the time of assay. Plasma pCRH levels were measured by specific double-antibody radioimmunoassays. Specific polyclonal rabbit antisera and the iodinated peptides were obtained from Peninsula Laboratories (Belmont, CA). Crossreactivities of $\mathrm{CRH}$ antiserum were $100 \%$ for human and rat $\mathrm{CRH}$ and none for the precursor of $\mathrm{CRH}(0 \%)$. Additional details of the radioimmunoassay procedure were described by Castro and colleagues (1989). The minimum detection limit for the assay was $10 \mathrm{pg} / \mathrm{mL}$ and the inter- and intra-assay variability was $3.7 \%$ and $5.2 \%$, respectively. Some samples at 19 weeks were below the detection limit when assayed in their original concentrations $(N=150)$. In these cases, samples were concentrated two or three times using a SpeedVac dry-down procedure described in Castro et al. (1989), until concentrations of pCRH were detectable by the assay. Values for these pCRH samples were then back calculated to represent picograms per milliliters $(\mathrm{pg} / \mathrm{mL})$ in their original concentrations.

Covariates-The following covariates were assessed: maternal age, household income, education (years in school), married or cohabiting with baby's father, parity (previous births vs. first birth), preterm birth, low birth weight, and smoking in pregnancy. These covariates were chosen because they have been identified by previous research as predictors of postpartum depression, social support, and $\mathrm{pCRH}$ or because they were plausible correlates. Birth records were obtained to assess preterm birth (defined as birth at less than 37 weeks' gestation; $0=$ term, $1=$ preterm) and low birth weight (defined as a birth weight under 2,500 g; $0=$ normal birth weight, $1=$ low birth weight). Women were asked to report their household incomes $(1=$ under $\$ 2,500 ; 2=\$ 2,501-\$ 5,000 ; 3=\$ 5,001-\$ 10,000$; and in $\$ 10,000$ increments thereafter, with a max of $\$ 100,000$ and over). We used married or cohabitating (instead of marital status alone) because preliminary analyses suggested that this combined variable was more predictive of perceived support from the baby's father than was marital status alone. ${ }^{1}$ Specifically, women who were unmarried/noncohabitating ( $n=$ 
49) reported significantly less support from the baby's father during pregnancy than women who were married/cohabitating $(n=129), F(2,208)=-0.55, S E=.20, p<.001$, or cohabitating/unmarried $(n=32), F(2,208)=-0.37, S E=.12, p<.01$. Support from the baby's father did not differ significantly between women who were married/cohabitating and those that were cohabitating/unmarried, $F(2,208)=-0.18, S E=.10, p=.072$. This variable was coded as a 1 for married and/or cohabiting and a 0 for unmarried and noncohabitating.

\section{Statistical analytic strategy}

Multilevel modeling was used to assess whether pCRH during pregnancy differed as a function of perceived social support or depressive symptoms. Multilevel modeling allows for the determination of between-person differences among within-person trajectories and offers advantages over other statistical tools for the evaluation of longitudinal data because it accounts for shared variance on within-individual measurements and can accommodate missing values (Raudenbush \& Bryk, 2002). Although previous studies of pCRH and depressive symptoms have used quadratic multilevel models because pCRH increases exponentially over the course of pregnancy (Yim et al., 2009), at least four observations are recommended to test quadratic growth curve models (Raudenbush \& Bryk, 2002). Because this data set had three observations of pCRH in pregnancy, an unrestricted model was used instead. Unrestricted models do not place any assumptions on the pattern of change in the data (because time is treated as a categorical variable) while still controlling for the shared variance between observations. Covariates were included in the multilevel models if they were significantly related to depressive symptoms (age, income, smoking in pregnancy), pCRH (preterm birth), or social support (education, income, married or cohabitating, parity, ethnicity; see Table 1 and Results for details). Ethnicity was coded to create a contrast between Whites (dummy code $=1$ ) and non-Whites (dummy code $=0$ ), given the pattern of significant ethnic differences that emerged in the analyses.

Preacher and Hayes's (2008) bootstrapping procedure was followed to test for mediation. For the covariate analysis, Pearson's correlations were used in cases of two continuous variables; Spearman's rank correlations were used in cases of ordinal variables (e.g., income); $t$ tests were used to identify significant differences as a function of dichotomous categorical variables; and analyses of variance were used in the case of categorical variables with more than two levels (e.g., ethnicity).

All analyses were conducted in SPSS 18, including multilevel modeling using the "mixed" function. Covariates were entered into multilevel models as time-unvarying/fixed predictors. Shared variance of within-subject pCRH levels were accounted for with an autoregressive error structure using the "repeated" random effects function. All continuous predictors were $Z$ scored ([participant's raw score - population mean] / population standard deviation) before being entered into multilevel models so that a one-unit increase in the coefficient would reflect a one-standard deviation increase in the outcome variable.

\footnotetext{
${ }^{1}$ Not to say that there were no significant differences between the married/cohabitating women versus the cohabitating/unmarried women. Married/cohabiting and cohabitating/unmarried women did differ in some ways: Married/cohabitating women had higher incomes, were more likely to be White, had more education, and had fewer children living in the home compared to cohabitating/ unmarried women. There were no significant differences between the married/cohabitating and cohabitating/unmarried groups in terms of our key variables of interest (social support, placental corticotropin-releasing hormone, or depressive symptoms); therefore, these groups were combined in our analysis.
} 


\section{Results}

pCRH increased significantly over time, $F(1,2)=79,111.27, p<.001$. The average pCRH values at T1, T2, and T3 were $8.4(S D=3.3, n=181), 248.25(S D=34.1, n=163)$, and $846.1(S D=35.4, n=181)$, respectively. The average level of perceived social support from family was $3.27(S D=0.60)$ and from the baby's father was $3.34(S D=0.60)$ on a 4-point scale. The average BDI sum score was $7.39(S D=1.02)$ in pregnancy and $6.19(S D=0.98)$ in the postpartum. In pregnancy, 26\% ( $n=54$ of 210) of our sample met the clinical cutoff for mild depressive symptomatology (BDI score $\geq 10)$, and $4 \%(n=8$ of 210 ) met the cutoff for moderate to severe depressive symptomatology (BDI score 219 ). In the postpartum, $19 \%$ ( $n=39$ of 210) met the cutoff for mild depressive symptomatology, and 2\% ( $n=5$ of 210) met the cutoff for moderate or severe depressive symptomatology.

\section{Bivariate effects}

Means and standard deviations of demographic and obstetric variables are presented in Table 1, along with results of bivariate tests showing how these variables related to depressive symptoms, pCRH, and social support. There were several significant bivariate associations between the covariates assessed in this study and the key variables of interest. Women with more depressive symptoms in pregnancy tended to be younger, have lower household incomes, and be more likely to smoke during pregnancy than women with fewer depressive symptoms in pregnancy. In contrast, postpartum depressive symptoms were not significantly related to any covariate tested. In terms of pCRH, women who delivered preterm had significantly higher pCRH at all three time points, consistent with previous reports (Hobel et al., 1999; Korebrits et al., 1998; McLean \& Smith, 1999). In addition, there was a trend for women who smoked during pregnancy to have higher pCRH at T2. Social support from the baby's father and from family was positively associated with household income and being a first-time mother. Women who reported more support from the baby's father tended to have higher levels of education than women reporting less support from the baby's father. As would be expected, women who were married or living with the baby's father reported considerably more support from the baby's father than women who were not married or cohabitating with the father. Ethnic differences in support emerged as well, with non-Hispanic White women reporting significantly more support from the baby's father than Latina/Hispanic or African American/Black women. However, there were no ethnic differences in levels of family support reported.

Bivariate correlations between depressive symptoms, pCRH, and support variables are presented in Table 2. Women who perceived more support from the baby's father also tended to report more support from family $(r=.23, p<.05)$. Depressive symptoms in pregnancy and postpartum were also positively correlated $(r=.47, p<.001)$, as were pCRH levels at the three time points $(r=.16-.46, p<.09-.01)$. Women with higher perceived support from family and the baby's father reported significantly lower levels of depressive symptoms in pregnancy and the postpartum. In addition, women who reported higher perceived support from family had significantly lower levels of pCRH at T3 in pregnancy. Father support was not significantly associated with $\mathrm{pCRH}$ at any time point. Women with higher pCRH at T3 were more likely to report depressive symptoms postpartum. pCRH was not related to depressive symptoms in pregnancy.

\section{pCRH and depressive symptoms}

There was not a significant interaction between time and prenatal depressive symptoms ( $p$ $=.39$ ). Likewise, the interactions between time and the cutoff for mild prenatal depressive symptoms (BDI $\geq 10$ ) and moderate to severe prenatal depressive symptoms (BDI $\geq 19$ ) 
were not significant $(p>.13)$. The relationship between prenatal depressive symptoms and pCRH remained nonsignificant when covariates were removed from the model.

The interaction between time and postpartum depressive symptoms was significant, $F(1,3)$ $=3.73, p<.05$. Women who had greater increases in pCRH from T2 to T3 (coeff $=5.61, S E$ $=2.03, d f=505, t=-2.76, p<.01)$ reported more depressive symptoms postpartum than women with less dramatic increases in pCRH between $\mathrm{T} 2$ and T3. In addition, women who had higher mean levels of pCRH at T3 (coeff $=5.61, S E=2.02, d f=504, t=2.76, p<.01)$ reported more depressive symptoms postpartum than women with lower pCRH at T3. Levels of $\mathrm{pCRH}$ at $\mathrm{T} 1$ and $\mathrm{T} 2$ and changes in $\mathrm{pCRH}$ from $\mathrm{T} 1$ to $\mathrm{T} 2$ were not significantly related to postpartum depressive symptoms.

The interaction between time and postpartum depressive symptoms remained significant even when prenatal depressive symptoms were included in the model, $F(1,3)=3.64, p<$. 05. Again, greater increases in pCRH from T2 to T3 ( coeff $=5.30, S E=2.21, d f=494, t=$ 2.50, $p<.05$ ) and higher absolute levels at T3 (coeff $=5.30, S E=2.13, d f=490, t=2.49, p$ $<.05)$ predicted more depressive symptoms postpartum above and beyond those reported in pregnancy.

Next, the BDI cutoff scores for mild (BDI $\geq 10$ ) and moderate to severe (BDI $\geq 19$ ) depression were used to predict pCRH trajectories during pregnancy. The interaction between time and the mild cutoff was nonsignificant $(p=.17)$; however, there was a significant interaction between time and the moderate to severe cutoff, $F(3,474)=2.71, p$ $<.05$. Again, women who scored above the moderate to severe cutoff postpartum had greater increases in pCRH from T2 to T3 ( coeff $=28.23, S E=12.43, d f=506, t=2.27, p$ $<.025$ ) and higher levels of pCRH at T3 (coeff $=28.27, S E=12.42, d f=505, t=2.27, p<$. $025)$ than women who scored below the depression cutoff. Differences in levels of pCRH were not significantly different as a function of BDI scores greater than or equal to 19 at T1 or at $\mathrm{T} 2$ or as a function of change in $\mathrm{pCRH}$ from $\mathrm{T} 1$ to $\mathrm{T} 2$.

\section{pCRH and social support}

There was a significant interaction between social support from family and $\mathrm{pCRH}$ trajectories during pregnancy, $F(1,3)=4.20, p<.01$. Women who had higher social support from family during pregnancy had less dramatic increases in pCRH during pregnancy from T2 to T3 (coeff $=-9.14, S E=2.60, d f=503, t=3.52, p<.001)$ and lower absolute levels at $\mathrm{T} 3$ (coeff $=-9.16, S E=2.60, d f=503, t=3.50, p<.01$ ) during pregnancy than women with less support from family during pregnancy. Differences in levels of pCRH were not significantly different at $\mathrm{T} 1$ to $\mathrm{T} 2$ or as a function of change in $\mathrm{pCRH}$ from $\mathrm{T} 1$ to $\mathrm{T} 2$ as a function of family support (all $p<.41$ ).

There was not a significant interaction between social support from the baby's father and time, $F(3,358)=0.53, p=.66$. Marital status or cohabitation with the baby's father did not moderate the effect of baby's father support on pCRH. Likewise, the interaction between support from the baby's father and time was not significant when only women who were married or cohabitating with the baby's father were included in the sample ( $n=49$ removed, $n=161$ remained).

\section{Mediation analysis}

Because more drastic increases in pCRH in late pregnancy emerged as a significant risk factor for postpartum depressive symptoms and because support from family appeared to be protective against these increases, a mediation analysis was conducted to test whether social support was protective against postpartum depressive symptoms through its effects on changes in pCRH during pregnancy. A change score was created in the subset of 137 
women who had the relevant pCRH data (T3 pCRH - T2 pCRH); higher scores reflected greater increases in pCRH from $\mathrm{T} 2$ to $\mathrm{T} 3$. Covariates were also included in the model, along with $\mathrm{T} 2 \mathrm{pCRH}$ because it is recommended to control for baseline levels when using change scores (Vickers \& Altman, 2001).

Regressions revealed that more social support from family at $\mathrm{T} 2$ predicted more gradual increases in pCRH from T2 to T3 $(\beta=-.24, S E=.08, p<.01)$ and fewer postpartum depressive symptoms $(\beta=-.24, S E=.12, p<.05)$. Simultaneous regression of changes in pCRH from $\mathrm{T} 2$ to $\mathrm{T} 3$ on postpartum depressive symptoms and family support suggested that changes in pCRH fully mediated the effect of family social support on postpartum depressive symptoms (Fig. 1). Specifically, the effect of family support on postpartum depressive symptoms was no longer significant when changes in pCRH from T2 to T3 were included in the model $(\beta=-.18, S E=.12, p>.10)$, whereas $\mathrm{pCRH}$ remained a significant predictor of depressive symptoms postpartum $(\beta=.24, S E=.12, p<.05)$. A bootstrap test with 10,000 replications indicated a significant indirect effect of family support on postpartum depressive symptoms via changes in pCRH from T2 to T3 (95\% confidence interval $=-0.15,-0.01$ ).

\section{Discussion}

The findings in this study of 210 women from diverse socioeconomic and ethnic/racial backgrounds were consistent with the hypothesis that perceived family social support during pregnancy protects women against postpartum depression by dampening maternal pCRH trajectories during pregnancy. Women who perceived more support from their families during pregnancy evinced slower rises in $\mathrm{pCRH}$ in late pregnancy compared to women who perceived themselves as possessing less family support. In addition, women who displayed attenuated increases in pCRH in late pregnancy reported fewer depressive symptoms postpartum than women who had larger increases in pCRH, thus replicating the results of Yim and colleagues (2009) with a larger, more ethnically diverse sample. This study also afforded an opportunity to test whether changes in pCRH mediated the observed relationship between family support and postpartum depressive symptoms. Consistent with predictions, changes in pCRH from T2 to T3 in pregnancy mediated the relationship between social support and depressive symptoms postpartum. These results represent an advance in our understanding in several ways.

A first way in which this study advances our understanding is that it independently replicates the somewhat contested association between pCRH and depressive symptoms postpartum. There are notable similarities between the results of this study and those of Yim and colleagues (2009) that may have contributed to the ability to replicate. Both studies found that more dramatic increases in pCRH from mid- to late pregnancy and higher absolute levels of pCRH in late pregnancy (37 weeks) were predictive of depressive symptoms postpartum, although the previous study found that high levels of pCRH emerged earlier in pregnancy than in the current study. Relatedly, Rich-Edwards and colleagues (2008) found higher levels of pCRH at 27 weeks in women with prenatal depressive symptoms but did not find an association between pCRH at 27 weeks' gestation and depressive symptoms 6 months after delivery; however, 6 months may have been too late to detect the effects of stress hormones in pregnancy on depressive symptoms postpartum (Halbreich, 2005). Of note is the fact that two studies found negative (rather than positive) associations between pCRH and prenatal depressive symptoms (Meltzer-Brody et al., 2011; Susman et al., 1999). Past results involving both negative and positive associations between pCRH and depressive symptoms may reflect the heterogeneity of depression itself (Diagnostic and Statistical Manual of Mental Disorders, fourth edition). Indeed, melancholic depression — characterized by high anxiety, appetite loss, and insomnia—is associated with 
hyperactive $\mathrm{CRH}$ activity in the nonpregnant state, whereas atypical depressioncharacterized by lethargy, fatigue, and insomnia-is associated with decreased CRH production (see Gold \& Chrousos, 2002, for a review). Future studies of pCRH and maternal depression might take these two subtypes of depression into account through the use of structured clinical interviews. Alternatively, inconsistencies in the literature could be due to (a) the numerous differences between studies in the timing of the assessments of pCRH or depressive symptoms, (b) the specific measures of depression used, or (c) variations in assays used to measure pCRH. For example, both Yim and colleagues (2009) and the current study found that accelerated pCRH trajectories in the latter half of pregnancy were predictive of postpartum depressive symptoms, whereas several works reporting null results had only one assessment of pCRH during midpregnancy (Rich-Edwards et al., 2008; Susman et al., 1999). Additional replication attempts are needed using comparable methods, especially given the unique opportunity that pregnancy affords to measure CRH in blood and the fact that the HPA axis is implicated in the etiology of depression in the nonpregnant state (see Ehlert et al., 2001; Pariante \& Lightman, 2008, for reviews).

A second advance in our understanding afforded by this work is the demonstration that family support was linked to maternal stress physiology. Women with higher levels of family support had slower increases in pCRH in late pregnancy and lower absolute levels of pCRH at 38 weeks' gestation. This finding suggests that family support may influence birth outcomes because pCRH has been implicated in preterm birth. pCRH has been termed the "placental clock" (McLean et al., 1995) in light of consistent evidence that its trajectory over the course of pregnancy reliably predicts the timing of delivery (Hobel et al., 1999;

Korebrits et al., 1998; Leung et al., 2001; McLean \& Smith, 1999). Women whose levels of pCRH increase more quickly during pregnancy are at greater risk of giving birth prematurely (see Erickson et al., 2001, for a review). Indeed, women who gave birth preterm in the BIPS sample had higher levels of pCRH throughout pregnancy (cf. Hobel et al., 1999; Mancuso, Dunkel Schetter, Rini, Roesch, \& Hobel, 2004). The results of this study imply that family support in pregnancy may protect against preterm birth by slowing pCRH trajectories. pCRH has also been implicated in the development of the fetal brain, and it plays a role in programming the infant's stress regulation systems (see Sandman \& Glynn, 2009 , for a review). Infants exposed to lower levels of pCRH at 25 weeks' gestation exhibit enhanced neurological maturity in response to stimulation in utero (Class et al., 2008) and less fear and distress at 2 months of age (Davis et al., 2005) than infants exposed to higher levels of pCRH. Given this, it is possible that social support from family could have positive influences on infant stress regulation and development through its influence on pCRH during pregnancy. Why family support rather than father support predicted changes in pCRH is unclear. It is possible that pregnancy represents a context in which family support, especially from the women's mother or grandmother, is especially palliative. It is noteworthy, however, that father support was significantly and inversely correlated with depressive symptoms during pregnancy in this study, with a similar trend emerging postpartum. These facts suggest that father support may exert its protective effects against maternal depression earlier than 19 weeks or through other biological or behavioral pathways not explored in this study. Future studies might investigate the role of specific family support relationships more closely and test whether father support exerts its protective effects though other biological stress or immune pathways in pregnancy (Tanner Stapleton et al., 2012).

This study benefited from a number of strengths - most notably, a prospective design and a relatively large, ethnically and socioeconomically diverse sample. However, it is also important to acknowledge a few limitations. First, mediation models cannot establish causality. Nevertheless, the time course that we observed was consistent with the causal explanation that we have advanced; social support at $\mathrm{T} 2$ predicted changes in $\mathrm{pCRH}$ 
between $\mathrm{T} 2$ and $\mathrm{T} 3$, which predicted depressive symptoms at $\mathrm{T} 4$, and the indirect relationship accounted for a significant amount of the variance of the support-depression association. The second limitation is that self-report measures of depression were used, whereas diagnostic interviews would be preferable. Follow-up studies can attempt to replicate the present findings using diagnostic tools such as the Structured Clinical Interview for DSM-IV.

The present results suggest many new avenues for future research. First, these results suggest possible translational opportunities through interventions to increase social support from family members to protect against postpartum depression. Though not a new idea, it may now have a novel impetus. For example, this study supports the design of randomized controlled trials with random assignment to family support enhancement or control conditions while measuring stress hormones throughout pregnancy as possible mediators. Previous randomized controlled trials designed to enhance social support during pregnancy have yielded modest or null effects on postpartum depression risk (see Clatworthy, 2012, for a review; Brugha et al., 2000; Matthey, Kavanagh, Howie, Barnett, \& Charles, 2004; Stamp, Williams, \& Crowther, 1995). However, these studies have targeted only father or peer support, whereas our results indicate that better results might be found by targeting the family, at least for women similar to our sample in terms of demographics. This research also suggests that the timing of any support intervention could be critical, with earlier intercessions being the most effective. Previous randomized control trials to enhance support were applied in late pregnancy, usually coinciding with birth preparation classes taken a few weeks before birth. Our results suggest that this may be too late in pregnancy to modify the increases in pCRH from 29 to 37 weeks' gestation found to reduce the likelihood of postpartum depression in this study. Second, a next generation of rigorously designed longitudinal observational studies are needed to replicate and extend these results. Those investigations should assess women at least four times during pregnancy to fully capture the exponential increases of pCRH and should include detailed measures of perceived support measured prospectively along with repeated assessments of depressive symptoms. Bringing theory and state-of-the-art social support measures (Rini \& Dunkel Schetter, 2010; Uchino, 2009) to bear in this area would also improve any future experimental or observational research. Furthermore, additional psychological resources besides social support, such as optimism, coping skills, and positive affect, could be examined, given that these resilience resources may be beneficial in warding off symptoms of postpartum depression by modulating stress hormones during pregnancy (Dunkel Schetter \& Dolbier, 2011; Zautra, Hall, Murray, \& the Resilience Solution Group, 2008).

Conceptually, this study bridges the traditional divide between biological and psychological predictors of postpartum depression. The prevailing research on the psychological contributors to physical health or birth outcomes stresses the importance of uncovering the biological mechanisms underlying these relationships (e.g., Mancuso et al., 2004; Miller, Chen, \& Cole, 2009). In contrast, models of psychological contributors to mental health outcomes often do not directly test physiological mediation. The underlying assumption that relationships between two psychological phenomena, such as the relationship between social support and depressive symptoms, could be adequately characterized without recourse to biology overlooks the fundamental reality that psychological processes are instantiated through biological processes. In the case of social support, the social environment has to "get under the skin" to protect women against a complex mental health disorder such as postpartum depression. If this materialistic premise is taken seriously, then clinical psychological science should not stop at detecting associations between psychological risk factors and mental illness but instead strive toward psychobiological integration in research and theory to formulate translational efforts. 


\section{Acknowledgments}

The study was supported by National Institute of Child Health and Human Development Grants R01 HD29553 and R01 HD28413 to Calvin J. Hobel and Christine Dunkel Schetter. Jennifer Hahn-Holbrook was supported by the MH15750 training grant fellowship during this work. Calvin J. Hobel was supported by the Miriam Jacobs Chair in Maternal Fetal Medicine during this work. We thank Heidi Kane for help with data analysis.

\section{References}

American Psychiatric Association. Diagnostic and Statistical Manual of Mental Disorders. 4th ed.. Washington DC: Author; 1994.

Arborelius L, Owens MJ, Plotsky PM, Nemeroff CB. The role of corticotropin-releasing factor in depression and anxiety disorders. Journal of Endocrinology. 1999; 160:1-12. [PubMed: 9854171]

Beck AX, Ward CH, Mendelson M, Mock J, Erbaugh J. An inventory for measuring depression. Archives of General Psychiatry. 1961; 4:561-571. [PubMed: 13688369]

Beck CT. A meta-analysis of predictors of postpartum depression. Nursing Research. 1996; 45:297303. [PubMed: 8831657]

Beck CT. Predictors of postpartum depression: An update. Nursing Research. 2001; 50:275-285. [PubMed: 11570712]

Bloch M, Daly RC, Rubinow DR. Endocrine factors in the etiology of postpartum depression. Comprehensive Psychiatry. 2003; 44:234-246. [PubMed: 12764712]

Brugha T, Wheatley S, Taub N, Culverwell A, Friedman T, Kirwan P, Shapiro D. Pragmatic randomized trial of antenatal intervention to prevent post-natal depression by reducing psychosocial risk factors. Psychological Medicine. 2000; 30:1273-1281. [PubMed: 11097068]

Bumberry W, Oliver JM, McClure JN. Validation of the Beck Depression Inventory in a university population using psychiatric estimate as the criterion. Journal of Consulting and Clinical Psychology. 1978; 46:150-155.

Campbell EA, Linton EA, Wolfe CDA, Scraggs PR, Jones MT, Lowry PJ. Plasma corticotropinreleasing hormone during pregnancy and partition. Journal of Clinical Metabolism. 1987; 63:10541059.

Castro LC, Arora C, Parvez S, Parvez H, Valenzuela G, Hobel CJ. Plasma atrial natriuretic peptide levels during the rat estrous cycle, pregnancy, and puerperium. American Journal of Obstetrics and Gynecology. 1989; 160:15-19. [PubMed: 2521423]

Chrousos GP, Torpy DJ, Gold PW. Interactions between the hypothalamic-pituitary-adrenal axis and the female reproductive system: Clinical implications. Annals of Internal Medicine. 1998; 129:229-240. [PubMed: 9696732]

Class QA, Buss C, Davis EP, Gierczak M, Pattillo C, Chicz-DeMet A, Sandman CA. Low levels of corticotropin-releasing hormone during early pregnancy are association with precocious maturation of the human fetus. Developmental Neuroscience. 2008; 30:419-426. [PubMed: 19127063]

Clatworthy J. The effectiveness of antenatal interventions to prevent postnatal depression in high-risk women. Journal of Affective Disorders. 2012; 137:25-34. [PubMed: 21514960]

Cohen S, Wills TA. Stress, social support, and the buffering hypothesis. Psychological Bulletin. 1985; 98:310-357. [PubMed: 3901065]

Collins NL, Dunkel-Schetter C, Lobel M, Scrimshaw SC. Social support in pregnancy: Psychosocial correlates of birth outcomes and postpartum depression. Journal of Personality and Social Psychology. 1993; 65:1243-1258. [PubMed: 8295121]

Davis EP, Glynn LM, Dunkel-Schetter C, Hobel C, Chicz-DeMet A, Sandman CA. Corticotropinreleasing hormone during pregnancy is associated with infant temperament. Developmental Neuroscience. 2005; 27:299-305. [PubMed: 16137987]

Dunkel Schetter C, Dolbier C. Resilience in the context of chronic stress and health in adults. Social and Personality Psychology Compass. 2011; 5:634-652.

Ehlert U, Gaab J, Heinrichs M. Psychoneuroendocrinological contributions to the etiology of depression, posttraumatic stress disorder, and stress-related bodily disorders: The role of the hypothalamus-pituitary-adrenal axis. Biology Psychology. 2001; 57:141-152. 
Erickson K, Thorsen P, Chrousos G, Grigoriadis DE, Khongsaly O, McGregor J, Schulkin J. Preterm birth: Associated neuroendocrine, medical, and behavioral risk factors. Journal of Clinical Endocrinology and Metabolism. 2001; 86:2544-2552. [PubMed: 11397853]

Evain-Brion D, Malassine A. Human placenta as an endocrine organ. Growth Hormone and IGF Research. 2003; 13:S34-S37. [PubMed: 12914725]

Feldman PJ, Dunkel-Schetter C, Sandman CA, Wadhwa PD. Maternal social support predicts birth weight and fetal growth in human pregnancy. Psychosomatic Medicine. 2000; 62:715-725. [PubMed: 11020102]

Field T. Postpartum depression effects on early interactions, parenting and safety practices: A review. Infant Behavior and Development. 2010; 33:1-6. [PubMed: 19962196]

Florio P, Zatelli MC, Reis FM, degli Uberti EC, Petraglia F. Corticotropin- releasing hormone: A diagnostic marker for behavioral and reproductive disorders? Frontiers in Bioscience. 2007; 12:551-560. [PubMed: 17127316]

Gaynes BN, Gavin N, Meltzer-Brody S, Lohr KN, Swinson T, Gartlehner G, Miller WC. Perinatal depression: Prevalence, screening accuracy, and screening outcomes. Evidence of Reproductive Technology Assessments. 2005; 119:1-8.

Gold PW, Chrousos GP. Organization of the stress system and its disregulation in melancholic depression: High vs low CRH/NE states. Molecular Psychiatry. 2002; 7:254-275. [PubMed: 11920153]

Gold PW, Licinio J, Wong ML, Chrousos GP. Corticotropin releasing hormone in the pathophysiology of melancholic and atypical depression and in the mechanism of action of antidepressant drugs. Annals of the New York Academy of Science. 1995; 771:716-729.

Gotlib, IH.; Cane, DB. Self-report assessment of depression and anxiety. In: Kendall, PC.; Watson, D., editors. Anxiety and depression: Distinctive and overlapping features. New York, NY: Academic Press; 1989. p. 131-169.

Gotlib IH, Whiffen VE, Mount JH, Milne K, Cordy NI. Prevalence rates and demographic characteristics associated with depression in pregnancy and the postpartum. Journal of Consultant and Clinical Psychology. 1989; 57:269-274.

Grace SL, Evindar A, Stewart DE. The effect of postpartum depression on child and cognitive development and behavior: A review and critical analysis of the literature. Archives of Women's Mental Health. 2003; 6:263-274.

Greenberg PE, Kessler RC, Birnbaum HG, Leong SA, Lowe SW, Berglund PA, Corey-Lisle PK. The economic burden of depression in the United States: How did it change between 1990 and 2000 ? Journal of Clinical Psychiatry. 2003; 64:1465-1475. [PubMed: 14728109]

Halbreich U. Postpartum disorders: Multiple interacting underlying mechanisms and risk factors. Journal of Affective Disorders. 2005; 88:1-7. [PubMed: 15996747]

Harris B, Lovett L, Newcombe RG, Read GF, Walker R, Riad-Fahmy D. Maternity blues and major endocrine changes: Cardiff puerperal mood and hormone study II. BMJ. 1994; 308:949-953. [PubMed: 8173402]

Hillerer KM, Neumann ID, Slattery DA. From stress to postpartum mood and anxiety disorders: How chronic peripartum stress can impair maternal adaptations. Neuroendocrinology. 2012; 95:22-38. [PubMed: 22042058]

Hobel C, Dunkel-Schetter C, Roesch S. Maternal stress as a signal to the fetus. Prenatal and Neonatal Medicine. 1998; 3:116-120.

Hobel CJ, Dunkel-Schetter C, Roesch SC, Castro LC, Arora CP. Maternal plasma corticotropinreleasing hormone associated with stress at 20 weeks gestation in pregnancies ending in preterm delivery. American Journal of Obstetrics and Gynecology. 1999; 180:S257-S263. [PubMed: 9914629]

Hochberg Z, Pacak K, Chrousos GP. Endocrine withdrawal syndromes. Endocrine Reviews. 2003; 24:523-538. [PubMed: 12920153]

Holcomb WL, Stone LS, Lustman PJ, Gavard JA, Mostello DJ. Screening for depression in pregnancy: Characteristics of the Beck Depression Inventory. Obstetrics and Gynecology. 1996; 88:10211025. [PubMed: 8942846] 
Holsboer F. Stress, hypercortisolism and corticosteroid receptors in depression: Implications for therapy. Journal of Affective Disorders. 2001; 62:77-91. [PubMed: 11172875]

Kasckow JW, Baker D, Geracioti TD Jr. Corticotropin-releasing hormone in depression and posttraumatic disorder. Peptides. 2001; 22:845-851. [PubMed: 11337099]

Kessler RC. Epidemiology of women and depression. Journal of Affective Disorders. 2003; 74:5-13. [PubMed: 12646294]

Korebrits C, Ramirez MM, Watson L, Brinkman E, Bocking AD, Challis JRC. Maternal corticotropinreleasing hormone is increased with impending preterm birth. Journal of Clinical Endocrinology and Metabolism. 1998; 83:1585-1591. [PubMed: 9589660]

Latendresse G, Ruiz RJ. Bioassay research methodology: Measuring CRH in pregnancy. Biological Research for Nursing. 2008; 10:54-62. [PubMed: 18647760]

Leung TN, Chung TKH, Madsen G, Lam PKW, Sahota D, Smith R. Rate of rise in maternal plasma corticotrophin-releasing hormone and its relation to gestational length. BJOG: An International Journal of Obstetrics and Gynaecology. 2001; 108:527-532. [PubMed: 11368140]

Logsdon MC, McBride AB. Social support and postpartum depression. Research in Nursing and Health. 1994; 17:449-457. [PubMed: 7972923]

Magiakou MA, Mastorakos G, Rabin D, Dubbert B, Gold PW, Chrousos GP. Hypothalamic corticotropin-releasing hormone suppression during the postpartum period: Implications for the increase in psychiatric manifestations at this time. Journal of Clinical Endocrinology and Metabolism. 1996; 81:1912-1917. [PubMed: 8626857]

Mancuso RA, Dunkel Schetter C, Rini CM, Roesch SC, Hobel CJ. Maternal prenatal anxiety and corticotropin-releasing hormone associated with timing of delivery. Psychosomatic Medicine. 2004; 66:762-769. [PubMed: 15385704]

Matthey S, Kavanagh DJ, Howie P, Barnett B, Charles M. Prevention of postnatal distress or depression: An evaluation of an intervention at preparation for parenthood classes. Journal of Affective Disorders. 2004; 79:113-126. [PubMed: 15023486]

McEwen BS. Stress, adaptation, and disease: Allostasis and allostatic load. Annals of the New York Academy of Sciences. 1998; 840:33-44. [PubMed: 9629234]

McLean M, Bisits A, Davies J, Woods R, Lowry P, Smith R. A placental clock controlling the length of human pregnancy. Nature Medicine. 1995; 1:460-463.

McLean M, Smith R. Corticotropin-releasing hormone in human pregnancy and parturition. Trends in Endocrinology and Metabolism. 1999; 10:174-178. [PubMed: 10370225]

McLean M, Thompson D, Zhang HP, Brinsmead M, Smith R. Corticotropin-releasing hormone and beta-endorphin in labour. European Journal of Endocrinology. 1994; 131:167-172. [PubMed: 8075786]

Meltzer-Brody S, Stuebe A, Dole N, Savitz D, Rubinow D, Thorp J. Elevated corticotropin releasing hormone (CRH) during pregnancy and risk of postpartum depression (PPD). Journal of Clinical Endocrinology and Metabolism. 2011; 96:40-47.

Miller G, Chen E, Cole SW. Health psychology: Developing biologically plausible models linking the social world and physical health. Annual Review of Psychology. 2009; 60:501-524.

Nestler EJ, Barrot M, DiLeone RJ, Eisch AJ, Gold SJ, Monteggia LM. Neurobiology of depression. Neuron. 2002; 34:13-25. [PubMed: 11931738]

Nobel RE. Depression in women. Metabolism. 2005; 54:49-52.

Norbeck JS, Anderson NJ. Life stress, social support, and anxiety in mid- and late-pregnancy among low income women. Research in Nursing and Health. 1989; 12:281-287. [PubMed: 2798948]

O'Hara MW. Postpartum depression: What we know. Journal of Clinical Psychology. 2009; 65:12581269. [PubMed: 19827112]

O'Hara MW, Schlechte JA, Lewis DA, Wright EJ. Prospective study of postpartum blues: Biologic and psychosocial factors. Archives of General Psychiatry. 1991; 48:801-806. [PubMed: 1929770]

O'Hara MW, Swain AM. Rates and risk of postpartum depression: A meta-analysis. International Review of Psychiatry. 1996; 8:37-54.

O'Hara MW, Zekoski EM, Philipps LH, Wright EJ. A controlled prospective study of postpartum mood disorders. Journal of Abnormal Psychology. 1990; 99:3-15. [PubMed: 2307763] 
Okano T, Nomura J. Endocrine study of the maternity blues. Progress in Neuropsychopharmacological Biological Psychiatry. 1992; 16:921-932.

Oliver JM, Simmons ME. Depression as measured by the DSM-III and the Beck Depression Inventory in an unselected adult population. Journal of Consulting and Clinical Psychology. 1984; 52:892898. [PubMed: 6501675]

Pariante CL, Lightman SL. The HPA axis in major depression: Classical theories and new developments. Trends in Neurosciences. 2008; 31:461-468.

Paulson JF, Dauber S, Leiferman JA. Individual and combined effects of postpartum depression in mothers and fathers on parenting behaviors. Pediatrics. 2006; 118:659-668. [PubMed: 16882821]

Petraglia F, Florio P, Nappi C, Genazzani AR. Peptide signaling in human placenta and membranes: Autocrine, paracrine, and endocrine mechanisms. Endocrine Reviews. 1996; 17:156-186. [PubMed: 8706630]

Pitt B. Maternity blues. British Journal of Psychiatry. 1973; 122:431-433. [PubMed: 4718277]

Preacher KJ, Hayes AF. Asymptotic and resampling strategies for assessing and comparing indirect effects in multiple mediator models. Behavior Research Methods. 2008; 40:879-891. [PubMed: 18697684]

Ramona M, Ferketich S. Stress and social support as predictors of anxiety and depression during pregnancy. Advances in Nursing Science. 1988; 10:26-39. [PubMed: 3124719]

Raudenbush, SW.; Bryk, AS. Hierarchical linear models: Applications and data analysis method. London, England: Sage; 2002.

Rich-Edwards JW, Mohllajee AP, Kleinman K, Hacker MR, Majzoub J, Wright RJ, Gillman MW. Elevated midpregnancy corticotropin-releasing hormone is associated with prenatal, but not postpartum, maternal depression. Journal of Clinical Endocrinology and Metabolism. 2008; 93:1946-1951. [PubMed: 18303075]

Rini, C.; Dunkel Schetter, C. The effectiveness of social support attempts in intimate relationships. In: Sullivan, K.; Davila, J., editors. Support processes in intimate relationships. New York, NY: Oxford University Press; 2010. p. 26-70.

Robertson E, Grace S, Wallington T, Stewart DE. Antenatal risk factors for postpartum depression: A synthesis of recent literature. General Hospital Psychiatry. 2004; 26:289-295. [PubMed: 15234824]

Ross LE, Sellers EM, Gilbert Evans SE, Romach MK. Mood changes during pregnancy and the postpartum period: Development of a biopsychosocial model. Acta Psychiatrica Scandinavica. 2004; 109:457-466. [PubMed: 15117291]

Roy A, Evers SE, Avison WR, Campbell MK. Higher zinc intake buffers the impact of stress on depressive symptoms in pregnancy. Nutrition Research. 2010; 30:695-704. [PubMed: 21056285]

Sandman C, Glynn LM. Corticotropin-releasing hormone (CRH) programs the fetal and maternal brain. Future Neurology. 2009; 4:257-261. [PubMed: 19680459]

Sasaki A, Liotta AS, Luckey MM, Margioris AN, Suda T, Krieger DT. Immunoreactive corticotropinreleasing factor is present in human maternal plasma during the third trimester of pregnancy. Journal of Clinical Endocrinology and Metabolism. 1984; 59:812-814. [PubMed: 6332823]

Shibasaki T, Odagiri E, Shizume K, Ling N. Corticotropin-releasing factor-like activity in human placental extracts. Journal of Clinical Endocrinology and Metabolism. 1982; 55:384-386. [PubMed: 6282924]

Smith R, Cubis J, Brinsmead M, Lewin T, Singh B, Owens P, Nolan M. Mood changes, obstetric experience and alterations in plasma cortisol, beta-endorphin and corticotrophin releasing hormone during pregnancy and the puerperium. Journal of Psychosomatic Research. 1990; 34:53-69. [PubMed: 2138227]

Smith SM, Vale WW. The role of the hypothalamic-pituitary-adrenal axis in neuroendocrine responses to stress. Dialogues in Clinical Neuroscience. 2006; 8:383-395. [PubMed: 17290797]

Stamp GE, Williams AS, Crowther CA. Evaluation of antenatal and postnatal support to overcome postnatal depression: A randomized controlled trial. Birth. 1995; 22:138-143. [PubMed: 7575861]

Stein G, Marsh A, Morton J. Mental symptoms, weight changes, and electrolyte excretion in the first post partum week. Journal of Psychosomatic Research. 1981; 25:395-408. [PubMed: 7328507] 
Susman EJ, Schmeelk KH, Worrall BK, Granger DA, Ponirakis A, Chrousos GP. Corticotropinreleasing hormone and cortisol: Longitudinal associations with depression and antisocial behavior in pregnant adolescents. Journal of the American Academy of Child Adolescent Psychiatry. 1999; 38:460-467. [PubMed: 10199119]

Tanner Stapleton LR, Dunkel Schetter C, Westling E, Rini C, Glynn LM, Hobel CJ, Sandman CA. Perceived partner support in pregnancy predicts lower maternal and infant distress. Journal of Family Psychology. 2012; 26:453-463. [PubMed: 22662772]

Turner, RJ.; Frankel, BG.; Levin, DM. Social support: Conceptualization, measurement, and implications for mental health. In: Greenley, JR., editor. Researching community and mental health. Vol. Vol. 3. Greenwich, CT: JAI Press; 1983. p. 67-111.

Turner RJ, Grindstaff CF, Phillips N. Social support and outcomes in teenage pregnancy. Journal of Health and Social Behavior. 1990; 31:43-57. [PubMed: 2313076]

Uchino BN. Understanding the links between social support and physical health: A life-span perspective with emphasis on the separability of perceived and received support. Perspectives on Psychological Science. 2009; 4:236-255.

Uchino BN, Cacioppo JT, Kiecolt-Glaser JK. The relationship between social support and physiological processes: A review with emphasis on underlying mechanisms and implications for health. Psychological Bulletin. 1996; 119:488-531. [PubMed: 8668748]

Vesga-Lopez O, Blanco C, Keyes K, Olfson M, Grant BF, Hasin DS. Psychiatric disorders in pregnant and post-partum women in the United States. Archives of General Psychiatry. 2008; 65:805-815. [PubMed: 18606953]

Vickers A, Altman DG. Analysing controlled trials with baseline and follow up measurements. BMJ. 2001; 323:1123-1124. [PubMed: 11701584]

Vitoratos N, Papatheodorou DC, Kalantaridou SN, Mastorakos G. "Reproductive" corticotropinreleasing hormone. Annals of the New York Academy of Sciences. 2006; 1092:310-318. [PubMed: 17308156]

Wisner KL, Peindel K, Hanusa BH. Relationship of psychiatric illness to childbearing status: A hospital-based epidemiological study. Journal of Affective Disorders. 1993; 28:39-50. [PubMed: 8326079]

Yim IS, Glynn LM, Dunkel-Schetter C, Hobel CJ, Chicz-DeMet A, Sandman CA. Risk of postpartum depressive symptoms with elevated corticotropin-releasing hormone in human pregnancy. Archives of General Psychiatry. 2009; 66:162-169. [PubMed: 19188538]

Zautra AJ, Hall JS, Murray KE. the Resilience Solutions Group. Resilience: A new integrative approach to health and mental health research. Health Psychology Review. 2008; 2:41-64.

Zelkowitz P, Milet TH. Postpartum psychiatric disorders: Their relationship to psychological adjustment and marital satisfaction in the spouses. Journal of Abnormal Psychology. 1996; 105:281-285. [PubMed: 8723010]

Zonana J, Gorman JM. The neurobiology of postpartum depression. CNS Spectrums. 2005; 10:792799. [PubMed: 16400241] 


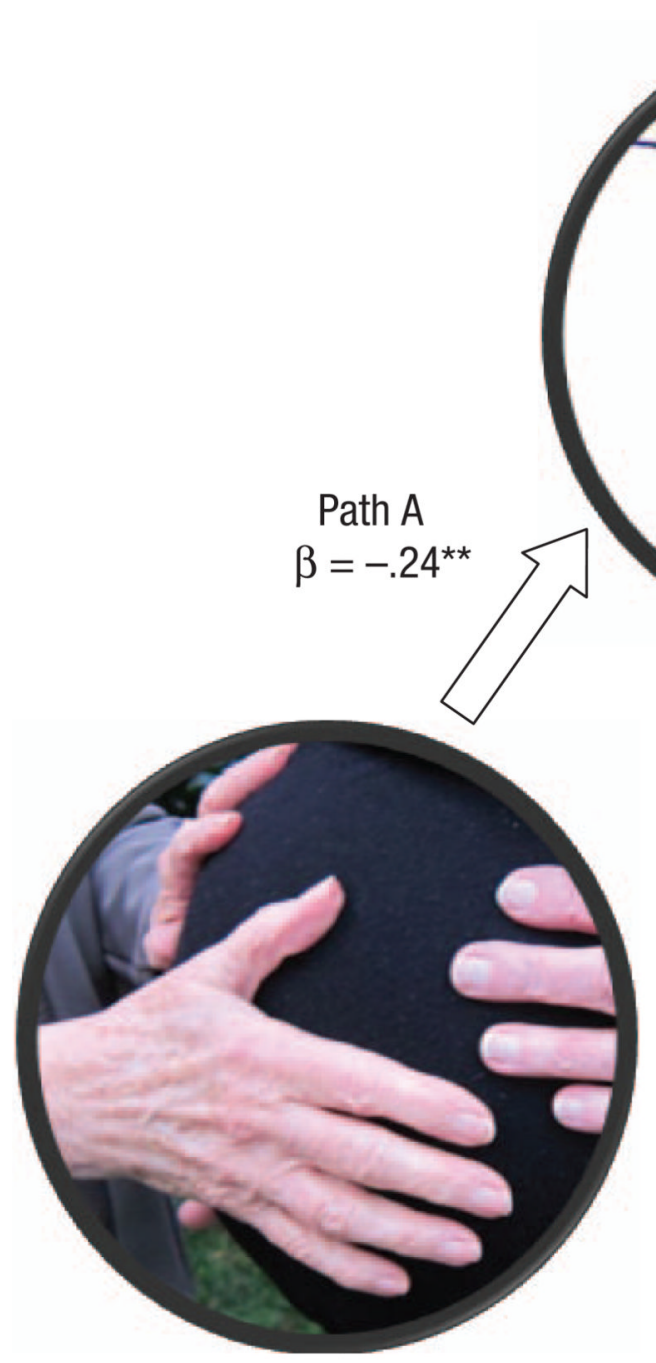

Family Support in

Pregnancy
Path B

$\beta=.24^{*}$

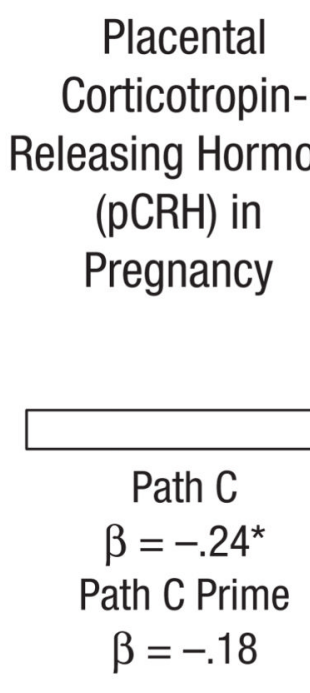

Fig. 1.

A mediation model of the association among perceived family support in pregnancy (T2), changes in placental corticotropin-releasing hormone (pCRH) during pregnancy (T2-T3), and postpartum depressive symptoms (T4). The indirect effect of family support on postpartum depression though changes in pCRH from T2 to T3 was significant (effect $=-$. $06, S E=.04,95 \%$ confidence interval $=-0.16,-0.01$ ), controlling for age, education, income, cohabitation with baby's father/marital status, parity, preterm, smoking in pregnancy, and ethnicity. ${ }^{*} p<.05 . * * p<.01$. 


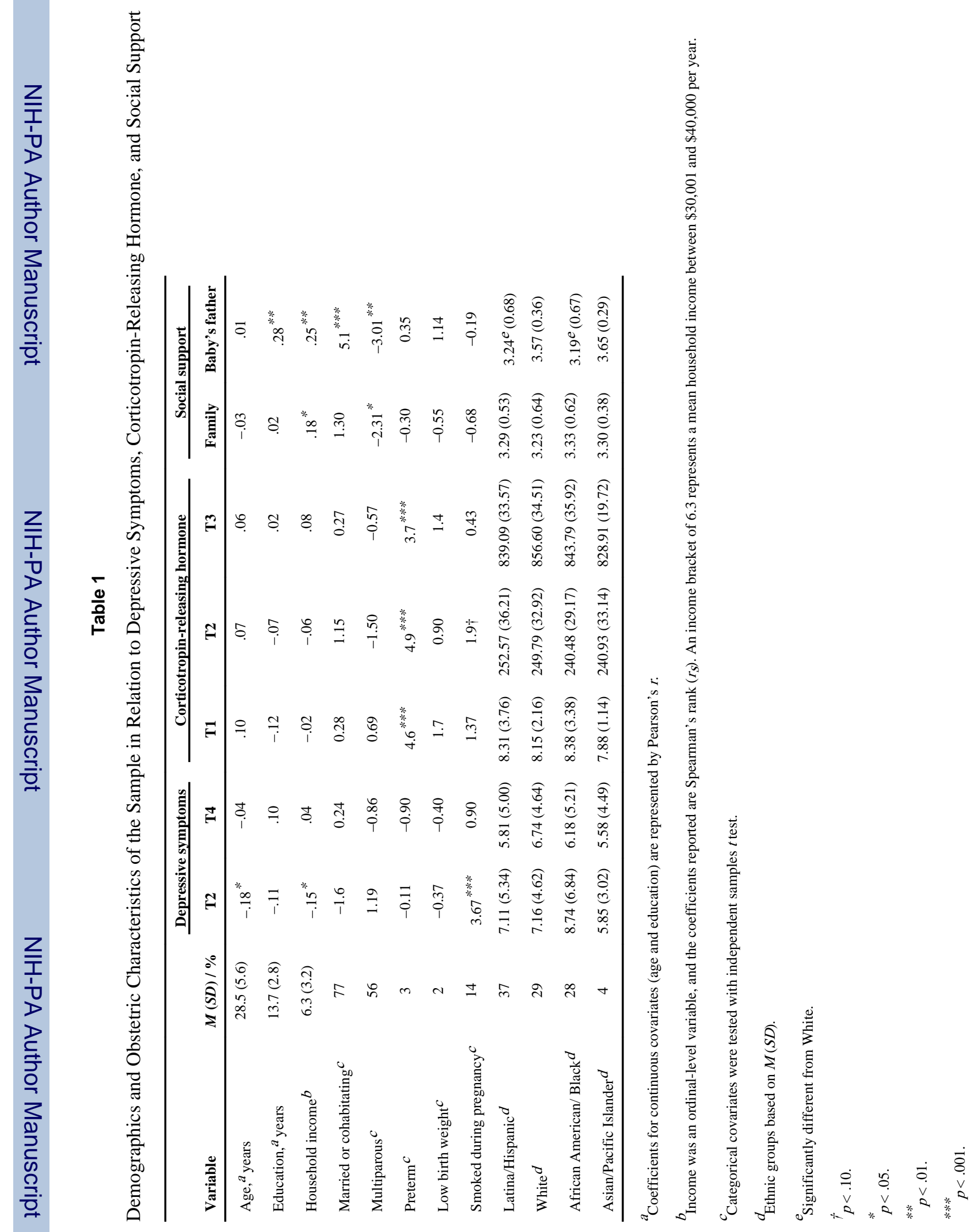




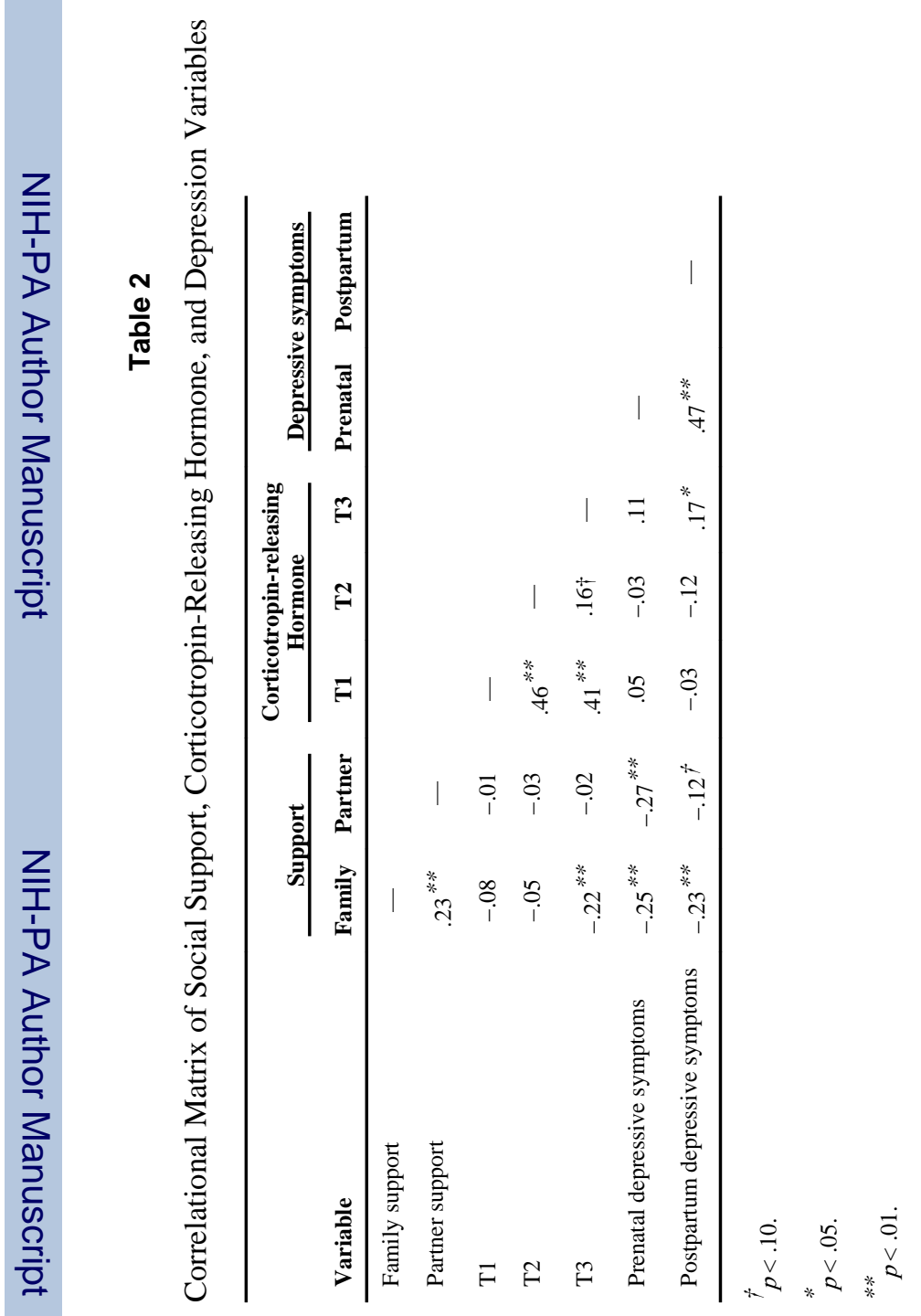

Clin Psychol Sci. Author manuscript; available in PMC 2014 January 01. 